Medicine, Kyungpook National University, Nuclear Medicine, Daegu, Korea, Rep. of (South Korea)

Background: Patients with the rheumatoid arthritis (RA) have an increased risk of cardiovascular disease (CVD) compared to general population. However there are insufficient modality to predict future CVD risk in RA.

Objectives: This study assessed whether splenic and arterial activity measured by positron emission tomography/ computed tomography (PET/CT) predict the risk of CVD thrombosis events beyond conventional risk factors in patients with RA.

Methods: We enrolled 84 patients with active RA who underwent fluorine-18-fluorodeoxyglucose (FDG) PET/CT and disease activity evaluation at the same time. CVD thrombosis events were independently evaluated, while blinded to activity of PET/CT, during follow up periods. FDG uptake by nuclear medicine physician was examined in the spleen and ascending aorta and blood pool activity of superior vena cava as SUV (standardized uptake values) and target-to-background-ratio (TBR) while blinded to CVD events.

Results: During follow-up periods, 19 patients developed CVD thrombosis events. Both splenic and arterial TBR were significantly increased in patients with subsequent CVD events compared to in patients without $(2.19 \pm 0.60$ vs $1.80 \pm 0.34, p$ $<0.013,1.72 \pm 0.22$ vs $1.57 \pm 0.22, \mathrm{p}<0.012$ ). Splenic TBR was associated with an increased risk of CVD events after adjustment for conventional CVD risk factors [hazard ratio $(\mathrm{HR}): 3.15$; $95 \%$ confidence interval $(\mathrm{Cl})$ : 1.46 to $6.79 ; \mathrm{p}=0.003$ ] Moreover, the association between splenic TBR and CVD events remained significant after adjustment for disease activity (HR: 3.00 ; $\mathrm{Cl}: 1.36$ to $6.63 ; p=0.007$ ) and after adjustment for arterial TBR (HR: 3.00 ; $\mathrm{Cl}: 1.36$ to $6.63 ; \mathrm{p}=0.007$ ).

Conclusion: Our results show splenic metabolic uptake in FDG-PET/CT in patients with RA provide information for subsequent CVD events beyond conventional risk factors.

REFERENCES

[1] Lee SJ, Jeong JH, Lee $\mathrm{CH}$, et al. Development and validation of an (18) F-fluorodeoxyglucose-positron emission tomography with computed tomography-based tool for the evaluation of joint counts and disease activity in patients with rheumatoid arthritis. Arthritis Rheumatol. 2019;71:1232-1240.

Disclosure of Interests: : None declared

DOI: 10.1136/annrheumdis-2021-eular.3574

\section{POS0097 JOINT INFLAMMATION TENDS TO RECUR IN THE SAME JOINTS DURING THE RHEUMATOID ARTHRITIS DISEASE COURSE}

S. Heckert ${ }^{1}$, S. A. Bergstra ${ }^{1}$, X. Matthijssen ${ }^{1}$, Y. Goekoop-Ruiterman ${ }^{2}$, F. Fodili ${ }^{3}$, C. Allaart ${ }^{1}$, T. Huizinga ${ }^{1} .{ }^{1}$ Leiden University Medical Center (LUMC), Rheumatology, Leiden, Netherlands; ${ }^{2}$ Hagaziekenhuis, Rheumatology, Den Haag, Netherlands; ${ }^{3}$ Reumazrg Z.-W. Eng., Roosendaal, Rheumatology, Roosendaal, Netherlands

Background: It is unknown whether in the disease course of rheumatoid arthritis (RA), inflammation recurs in the same joints over time or is more variable in joint locations. Joint involvement patterns over time might provide clues about the underlying mechanisms causing local joint inflammation.

Objectives: The aim of this study is to assess if local joint inflammation at presentation of RA tends to recur or persist in the same joints.

Methods: Data from the BeSt study were used, a treat-to-target (DAS $\leq 2.4)$ trial in newly diagnosed RA (ACR 1987 criteria) patients. During 10 years, for each patient 68 joints were assessed three-monthly ( 41 visits) by trained nurses for swelling (yes/no) and tenderness.

We analyzed the association between local joint swelling at baseline and later swelling of the same joint using a multilevel mixed-effects logistic regression model. Models were adjusted for joint location and for timepoint, with joints clustered within patients. A sensitivity analysis was done for the $25 \%$ most affected joints (MCP 1-3, PIP 2-3, wrists and MTP 2-4).

To investigate whether later swelling of a joint is predicted by baseline swelling of that same joint specifically, rather than by baseline swelling in general, a permutation test with 1000 permutations was performed. A p-value $<0.05$ indicates that joint swelling is better predicted by its baseline swelling than by baseline swelling of randomly selected other joints.

In a separate model, with an interaction term between baseline swelling and previous visit swelling (yes/no), we evaluated if the association between baseline swelling and later local swelling was influenced by whether later swelling was persistent (swelling at both the current and previous visit) or recurrent (swelling at current visit but not at the previous visit)

Results: The 508 patients had a median (IQR) follow-up duration of 10 (6-10) years. At baseline, 8,137/34,423 (24\%) assessed joints were scored as swollen. Baseline swelling was subsequently persistent in $21 \%$ of the joints with a median (IQR) duration of 1 (1-2) visit ( \pm 3 months after baseline). In addition, after resolution of initial swelling, swelling recurred at least once in $46 \%$ of the joints with baseline swelling. Baseline swelling was significantly associated with swelling in the same joint during follow-up (OR $2.37,95 \% \mathrm{Cl} 2.30-2.43$ ). A sensitivity analysis of the most affected joints showed similar results (OR 2.10 [95\% Cl 2.03-2.19]).
The permutation test showed a significant result with $p<0.001$, indicating that joint swelling is better predicted by baseline swelling of that same joint than by baseline swelling of other joints.

The association between baseline swelling and later local swelling was weaker in case of persistent swelling than in case of recurrent swelling (interaction term baseline swelling * swelling at previous timepoint 'yes': OR 0.80 [95\% Cl 0.75-0.85]). Conclusion: In newly diagnosed RA, over median 10 years of treatment to target DAS $\leq 2.4$, baseline swelling persisted in $21 \%$ of the joints, for median 3 months after baseline. Local recurrence after initial resolution occurred in $46 \%$ of the joints. Baseline joint swelling was significantly associated with local joint swelling during follow-up, even when taking into account the higher a priori chance of swelling in the joints that are most often affected, and joint swelling during follow-up was better predicted by baseline swelling of that particular joint than by baseline swelling of other joints. Local persistence and recurrence of joint swelling despite DAS $\leq 2.4$ steered treatment adjustments suggest that local joint conditions or even joint memory play a role in mechanisms of joint inflammation. Acknowledgements: We would like to thank all patients for their contribution as well as the rheumatologists who participated in the BeSt study group. We would also like to thank all other rheumatologists and trainee rheumatologists who enrolled patients in these studies, and all research nurses for their contributions Disclosure of Interests: Sascha Heckert: None declared, Sytske Anne Bergstra: None declared, Xanthe Matthijssen: None declared, Yvonne Goekoop-Ruiterman: None declared, F. Fodili: None declared, Cornelia Allaart Grant/research support from: The original BeSt study was supported by a government grant from the Dutch insurance companies, with additional funding from Schering-Plough B.V. and Janssen B.V., Thomas Huizinga: None declared DOI: 10.1136/annrheumdis-2021-eular.280

\section{POS0098 ARTHRITIS TO INFECTIOUS AND WOUND COMPLICATIONS AFTER TOTAL HIP AND KNEE ARTHROPLASTY}

A. Khramov ${ }^{1}$, M. Makarov ${ }^{1}$, S. Makarov ${ }^{1}$, S. Maglevaniy ${ }^{1}$, E. Naryshkin ${ }^{1}$, A. Dubinin ${ }^{1}$. 'V. A. Nasonova Research Institute of Rheumatology, Orthopaedic, Moscow, Russian Federation

Background: Surgical treatment of patients with rheumatoid arthritis (RA) is associated with an increased risk of complications. This is due to the presence of inflammation, many variants of the disease, reduced physical activity, severity of functional disorders, prolonged therapy with glucocorticoids, disease-modifying antirheumatic drugs (DMARDs) and biological DMARDs, osteoporosis, as well as activity of the underlying disease.

Objectives: to conduct a comparative analysis of the influence of RA activity levels on infectious complications (periprosthetic infection) and wound complications (poor healing, divergence, necrosis of the wound edges) after hip and knee arthroplasty in RA patients.

Methods: 1113 arthroplasties were analyzed in patients with RA, which were performed between 2002 and 2019. Of these, 649 total knee arthroplasties and 464 total hip arthroplasties were performed.

Results: Infectious complications after total hip and knee arthroplasty did not occur at 0 grade of disease activity (remission). At the I grade of activity, periprosthetic infections were detected with a frequency of $0.31 \%$, at the II grade $-0.89 \%$, and at the III level in $3.06 \%$ of cases.

Complications from the operative wound occurred in $0.91 \%$ of cases with I grade of activity, at II grade with a frequency of $5.68 \%$, and at III $-6.98 \%$. There were no cases of complications from the wound in patients with remission of RA.

Statistical analysis of the obtained data revealed a significantly higher number of complications in the group of RA patients $(p<0.005)$. During analyzing each type of complication, significant differences were also obtained $(p<0.005)$.

Conclusion: Risk of periprosthetic infection and complications from the wound is several times higher in patients with a high grade of RA activity. This means that performing arthroplasty, as well as other operations, in patients with high RA activity correlates to a high risk of complications.

Disclosure of Interests: None declared

DOI: 10.1136/annrheumdis-2021-eular.626

\section{POS0099 CLINICAL REMISSION IN RHEUMATOID ARTHRITIS PATIENTS TREATED WITH TOFACITINIB IS ASSOCIATED WITH LOW BASELINE EXPRESSION OF GENES RELATED TO ENERGY METABOLISM AND WITH CELLULAR CAPACITY OF THEIR UPREGULATION DURING FOLLOW-UP}

E. Tchetina ${ }^{1}$, A. Satybaldyev ${ }^{2}$, G. Markova ${ }^{1} .{ }^{1}$ Nasonova Research Institute of Rheumatology, Immunology and Molecular Biology, Moscow, Russian Federation; ${ }^{2}$ Nasonova Research Institute of Rheumatology, Early Arthritis, Moscow, Russian Federation 
Background: Rheumatoid arthritis (RA) is an autoimmune disease of unknown etiology, which is characterized by erosive arthritis (synovitis) and systemic inflammation. Tofacitinib (TFCN) is a small molecule Janus kinase (JAK) inhibitor that targets JAK1/JAK3. Identification of patients sensitive to TFCN before treatment could significantly improve therapy outcome. Presently it is not possible to predict TFCN efficacy in every patient while some patients are non-responsive to the drug that may produce adverse effects. TFCN function in RA patients has been recently associated with alterations in bioenergetics, mitochondrial function, and ATP production [1]. Therefore, we hypothesized that baseline metabolic status of RA patients prior to drug administration can predict the therapeutic outcome.

Objectives: To investigate the importance of baseline expression of genes involved in energy generation in RA patients, which could serve prognostic biomarkers for treatment response to tofacitinib.

Methods: Peripheral blood of 28 RA patients aged $52.2 \pm 15.6$ years old, average disease duration 3.5 years (range $0.6-19)$ treated with TFCN $(5-10 \mathrm{mg}$ twice a day) during three months and 26 healthy age-matched control subjects were examined. Clinical response was assessed by disease activity score (DAS28-ESR), serum levels of ACPA antibodies, rheumatoid factor $(R F), C$-reactive protein (CRP), and erythrocyte sedimentation rate (ESR). Clinical remission was assessed according to ACR criteria and DAS28 (DAS28 <2.6). Protein concentrations were measured using ELISA. Total RNA was isolated and used in gene expression studies performed with quantitative real-time RT-PCR.

Results: All of the patients were Steinbrocker's radiographic stage II-III at baseline. The majority of patients demonstrated erosive arthritis (23 out of 28), they were ACPA- (25 out of 28 ) and RF- (24 out of 28 ) positive. TFCN treatment significantly decreased the disease activity according to DAS28. At the end of the study the majority of patients demonstrated moderate disease activity (3.2< DAS28 $<5.1$ ), four patients retained high disease activity while 8 , attained remission (DAS28 <2.6). This was accompanied by significant decrease in CRP and the number of swollen and tender joints. ESR values were not changed significantly. Gene and protein expression analysis revealed that RA patients, which attained clinical remission after TFCN treatment demonstrated significantly lower baseline expression of genes associated with glycolysis (pyruvate kinase), oxidative phosphorylation (succinate dehydrogenase and uncoupling protein (UCP) 2) compared to other examined RA patients and control subjects. Moreover, these gene expressions increased in RA patients who attained clinical remission in the course of follow-up while in refractory for TFCN treatment patients these gene expressions were tending to downregulate.

Conclusion: Clinical remission attainment in RA patients treated with tofacitinib is associated with lower baseline expression of genes associated with energy generation pathways (pyruvate kinase, succinate dehydrogenase, and UCP2) compared to other examined subjects. Non-responsiveness to tofacitinib is accompanied by high baseline expression of genes related to glycolysis and oxidative phosphorylation compared to controls.

REFERENCES:

[1] McGarry et al. JAK/STAT Blockade Alters Synovial Bioenergetics, Mitochondrial Function, and Proinflammatory Mediators in Rheumatoid Arthritis. Arthritis Rheumatol, 2018; 70:1959.

Acknowledgements: Russian Ministry of Education and Science (Project No. AAAA-A19-11-9021190145-2 to EVT).

Disclosure of Interests: None declared

DOI: 10.1136/annrheumdis-2021-eular.837

\begin{tabular}{|l|l}
\hline POS0100 & VITAMIN D LEVEL IN RHEUMATOID ARTHRITIS \\
PATIENTS STARTING A BIOLOGIC DISEASE- \\
MODIFYING DRUG AND ITS CORRELATION WITH \\
DISEASE ACTIVITY AND RESPONSE TO TREATMENT
\end{tabular}

S. Garcia ${ }^{1}$, B. M. Fernandes ${ }^{1}$, F. Oliveira Pinheiro ${ }^{1}$, M. Rato ${ }^{1}$, D. Fonseca ${ }^{2}$, A. Martins ${ }^{1}$, D. Santos Oliveira ${ }^{1,3}$, F. R. Martins ${ }^{4}$, G. Terroso ${ }^{1}$, M. Bernardes ${ }^{1,5}$, L. Costa'. 'S ão João Universitary Hospital Center, Rheumatology, Porto, Portugal; ${ }^{2}$ Centro Hospitalar Vila Nova de Gaia / Espinho - Unit 1, Rheumatology, Vila Nova de Gaia, Portugal; ${ }^{3}$ Faculdade de Medicina da Universidade do Porto - FMUP, Center for Health Technology and Services Research (CINTESIS), Porto, Portugal; ${ }^{4}$ University Hospital Center of Algarve, Faro, Rheumatology, Faro, Portugal; ${ }^{5}$ Faculdade de Medicina da Universidade do Porto - FMUP, Rheumatology, Porto, Portugal

Background: Vitamin D, a fat soluble vitamin that is mainly involved in the regulation of calcium/phosphate metabolism, has a increasingly understood role in immunomodulatory activity, both in innate and adaptive immune system. In rheumatoid arthritis (RA), vitamin D showed to suppress the proliferation of synoviocytes and to reduce the production of proinfammatory cytokines, in vitro. (1) Recently the hypothesis has been raised that vitamin D has a negative association with RA activity. (2)
Objectives: This study aimed to evaluate the relationship between the 25-hydroxyvitamin $\mathrm{D}(25(\mathrm{OH})$ vitD) level, RA activity and response to a first biologic disease-modifying drug (bDMARD).

Methods: This is a longitudinal, retrospective study including consecutive patients with the diagnosis of RA followed at our rheumatology department. Demographic, clinical, and laboratorial data were collected from our national database at baseline, 6 and 12 months after initiation of a first bDMARD. Statis tical analysis was performed using SPSS 23.0. Correlations between variables were studied using Spearman correlation analysis and comparison between groups was performed using Wilcoxon and Kruskal-Wallis tests; $p<0.05$ was considered statistically significant.

Results: Mean age of patients $(n=236)$ was $51.5 \pm 11.2$ years old, $192(81.4 \%)$ were females with a median disease duration of 10.1 [4.7, 16.7] years. Seropositivity for anti-citrullinated protein antibodies was present in 192 (81.4\%) patients and for rheumatoid factor in 175 (74.2\%). The majority exhibited a very high or high disease activity at baseline (median DAS28 5.75 [4.99 - 6.63]) and $90 \%(n=212)$ of them were concomitantly using corticosteroids and/or other disease-modifying anti-rheumatic drugs (117 with methotrexate (MTX), 62 with leflunomide and 32 with sulfasalazine). Regarding bDMARD, 56.8\% ( $n=134)$ initiated an TNF alpha inhibitor.

After 6 and 12 months from a bDMARD initiation there was a significant reduction of ESR, CRP levels, TJCs, SJCs and DAS28 (all p-values $<0.001$ ), as expected. Median baseline serum 25(OH) vitD concentrations was $25.5[16.5,30.0] \mathrm{ng} /$ $\mathrm{ml}$; notably, $34.2 \%$ of our sample was affected by hypovitaminosis $\mathrm{D}$ at baseline $(25(\mathrm{OH}) \mathrm{vitD}<20 \mathrm{ng} / \mathrm{mL})$

Among our study population $42.5 \%$ patients were responders to first bDMARD (23.8\% good and $18.7 \%$ moderate responders) according to the EULAR response criteria. Disease remission (DAS28 $<2.6$ ) was achieved by $17.6 \%$ of patients.

The percentage of good responders was significantly lower in the subgroup of patients with hypovitaminosis D compared to subjects with normal $25(\mathrm{OH})$ vitamin $D$ levels at baseline $(p=0.002)$, as it was for the percentage of disease remission $(p=0.015)$.

The bivariate correlation analyses showed that $25(\mathrm{OH})$ vit $\mathrm{D}$ levels at baseline correlated with CRP levels and good response to RA treatment after 12 months (Spearman's coefficient $-0.201, p=0.028$; Spearman's coefficient 0.255 $p<0.019$, respectively). 25(OH) vit D levels at baseline, 6 and 12 months after bDMARD initiation did not correlate with age, BMI, ESV, number of tender or swollen joints, DAS28, HAQ or with SDAI or CDAI at 6 or 12 months of treatment Conclusion: In patients with RA, basal $25(\mathrm{OH})$ vit D levels correlated with response to a bDMARD. These results suggest a role of basal vitamin $D$ status in the prediction of disease evolution and support the hypothesis that vitamin D has an immunomodulatory potential.

\section{REFERENCES:}

[1] Huhtakangas JA, Veijola J, Turunen S et al. 1,25(OH)2D3 and calcipotriol, its hypocalcemic analog, exert a long-lasting anti-infammatory and anti-proliferative effect in synoviocytes cultured from patients with rheumatoid arthritis and osteoarthritis. J Steroid Biochem Mol Biol 2017; 173: 13- 22.

[2] Lee YH, Bae SC. Vitamin D level in rheumatoid arthritis and its correlation with the disease activity: a meta-analysis. Clin Exp Rheumatol. 2016 SepOct;34(5):827-833. Epub 2016 Apr 6. PMID: 27049238.

Disclosure of Interests: None declared

DOI: 10.1136/annrheumdis-2021-eular.1172

\section{SLE, Sjögren's and APS - clinical}

\section{\begin{tabular}{|l|l}
\hline POS0101 ADVERSE HEALTH-RELATED QUALITY OF \\
\hline
\end{tabular} LIFE OUTCOME DESPITE ADEQUATE CLINICAL RESPONSE TO TREATMENT IN SYSTEMIC LUPUS ERYTHEMATOSUS}

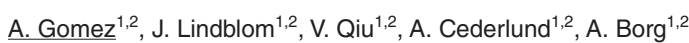

S. Emamikia ${ }^{1,2}$, Y. Enman ${ }^{1,2}$ J. Lampa ${ }^{1,2}$, I. Parodis ${ }^{1,2}{ }^{1}$ Karolinska Institutet,

Division of Rheumatology, Department of Medicine Solna, Stockholm, Sweden;

${ }^{2}$ Karolinska University Hospital, Department of Gastroenterology, Dermatology and Rheumatology, Stockholm, Sweden

Background: Despite improvements in medical care that have contributed to prolonged life expectancy for people living with systemic lupus erythematosus (SLE) over the past decades, they still suffer from substantial diminutions of health-related quality of life $(\mathrm{HRQoL})$ compared with the general population and with other chronic diseases.

Some studies have demonstrated that conventional synthetic and biological disease-modifying agents contribute to improvements in SLE patients' HRQOL, and responders to treatment have been shown to report greater improvements than non-responders. Although these observations are clinically relevant 